\title{
A case with GRIN2A mutation and its non-neurological manifestations
}

\author{
Soo Yeon Lee ${ }^{\oplus}$, So Yoon Jung ${ }^{\oplus}$, and Jeongho Lee ${ }^{* \oplus}$ \\ Department of Pediatrics, Soonchunhyang University Seoul Hospital, Seoul, Korea
}

\begin{abstract}
In epilepsy-aphasia spectrum (EAS) disorders, mutations in the glutamate receptor ionotropic $\mathrm{N}$-methyl-D-aspartate type subunit 2A (GRIN2A) have become important for screening the disease. Research into the phenotypic variability of several types of neurologic impairment involving these mutations is in progress. However, the non-neurological problems related to these mutations are poorly understood. EAS disorders usually have epileptic, cognitive, or behavioral manifestations. In this case report, we present a female patient with epilepsy, delay in expressive language and social development, and intellectual disability with low intelligence quotient and memory quotient, but normal motor development. Through genetic analysis, she was found to have a missense and a nonsense mutation in GRIN2A (c.1770A>C; p.Lys509Asn and c.3187G>T; p.Glu1063*, respectively) and we consider the nonsense mutation as 'pathogenic variant'. She was also discovered to have congenital hypothyroidism, growth hormone deficiency and Rathke's cleft cyst in the brain, which were previously unknown features of GRIN2A mutation. Our findings should widen understanding of the spectrum of GRIN2A phenotypes, and emphasize the need for more research into the association between GRIN2A mutations and non-neurologic clinical presentations.
\end{abstract}

Key words: Epilepsy, Intellectual disability, Congenital hypothyroidism.

\section{Introduction}

Epilepsy-aphasia spectrum (EAS) disorders present with seizures, abnormal electroencephalogram (EEG) results, usually in combined with cognitive and behavioral impairments [1]. Previously, these EAS disorders were thought to involve problems with the immune system, and high-dose steroids were usually used for treatment [2]. However, recent studies have investigated the genetic basis of the condition, and have identified mutations in GRIN2A, which encodes the $\alpha 2$ subunit of the $\mathrm{N}$ methyl-D-aspartate (NMDA) receptor, as a monogenic reason for EAS disorders $[2,3]$. The mutations have been found in at least $9 \%$ of patients with EAS disorders [2-4]. The mutation is inherited in autosomal dominant (AD) way and sometimes reported as a de-novo mutation $[5,6]$.

The NMDA receptor is a neurotransmitter-gated ion channel, comprised of two glycine-binding GluN1 subunits encoded by GRIN1 and two glutamate-binding GluN2 subunits encoded by GRIN2A-D [7]. The receptor is expressed throughout the brain and mediates the excitatory transmissions of brain functions. Consequentially, dysfunctions of the NMDA receptor can lead to various neurological diseases, such as epilepsy $[7,8]$. The effect of GRIN2A mutations on those NMDA receptors is considered to be a crucial link between the genetics and different epileptic and developmental features $[2,3]$.

The clinical spectrum of GRIN2A-related EAS is, however,

Received: 25 May 2020, Revised: 15 June 2020, Accepted: 15 June 2020, Published: 31 December 2020

${ }^{*}$ Corresponding author: Jeongho Lee, M.D., Ph.D. (iD https://orcid.org/0000-0003-4455-7540

Department of Pediatrics, Soonchunhyang University Seoul Hospital, 59 Daesagwan-ro, Yongsan-gu, Seoul 04401, Korea.

Tel: +82-2-709-9341, Fax: +82-2-709-9135, E-mail: ljh@schmc.ac.kr

Conflict of interest: The authors declare that they do not have any conflicts of interest.

(ㄷ) This is an open-access article distributed under the terms of the Creative Commons Attribution Non-Commercial License (http://creativecommons.org/licenses/by-nc/4.0/) which permits unrestricted non-commercial use, distribution, and reproduction in any medium, provided the original work is properly cited.

(c) Copyright 2020 by the Korean Society of Medical Genetics and Genomics 
much broader than has formerly apparent and no clear genotype-phenotype correlations have been identified. In particular, the relationship between the mutations and non-neurological symptoms has not yet been investigated. In this report, we present a case found to have GRIN2A mutations with both neurological and non-neurological problems, including congenital hypothyroidism leading to growth hormone $(\mathrm{GH})$ deficiency, and Rathke's cleft cyst. It is clear that mutations in GRIN2A have phenotypic effects beyond the brain.

\section{Case}

A female patient was born at 39 weeks of gestation by vaginal delivery, after a normal pregnancy with no birth injury, she was the second child in her family. At birth, her height and weight were within normal limits.

She was first diagnosed as suffering from congenital hypothyroidism. When she was three-weeks old, she was taken to hospital because of neonatal jaundice; her total serum bilirubin was high, at $17.8 \mathrm{mg} / \mathrm{dL}$. Photo therapy was administered, and total bilirubin returned to normal. A thyroid function test revealed a slightly elevated level of thyroid stimulating hormone (TSH). None of her family members have thyroid related disease, and her initial TSH level measured during neonatal screening test was normal. When she was one-month-old and weighting $5.6 \mathrm{~kg}$, we followed up her thyroid hormone levels and found that her TSH was still above $5 \mu \mathrm{IU} / \mathrm{mL}$ (TSH $8.61 \mu \mathrm{lU} / \mathrm{mL}$, free T4 $1.42 \mathrm{ng} / \mathrm{dL}$, T3 $127.94 \mathrm{ng} / \mathrm{dL}$ ). Thyroid ultrasonography showed a normal position and appearance of the thyroid gland, but Tc$99 \mathrm{~m}$ thyroid scan uptake was low at $0.8 \%$ (normal range, $0.3-$ $3.0 \%)$. Since the patient needed thyroid hormone replacement, we started to treat her with levothyroxine ( $30 \mathrm{mcg} /$ day). After three years of medication, we re-evaluated her thyroid function, and the levels of TSH and free T4 were normal, so treatment was discontinued. However, two years later, thyroid function tests showed low free T4 level at $0.58 \mathrm{ng} / \mathrm{dL}$ and she was restarted on levothyroxine. This condition was distinct from transient primary congenital hypothyroidism, which usually recovers to euthyroidism within the first years of life.

Epilepsy was another feature that needed treatment. Although the results of her neonatal screening test were all normal, at four years old, a febrile seizure event occurred, with generalized tonic movement, and after then, unprovoked seizure events followed at least twice a year. Those events were

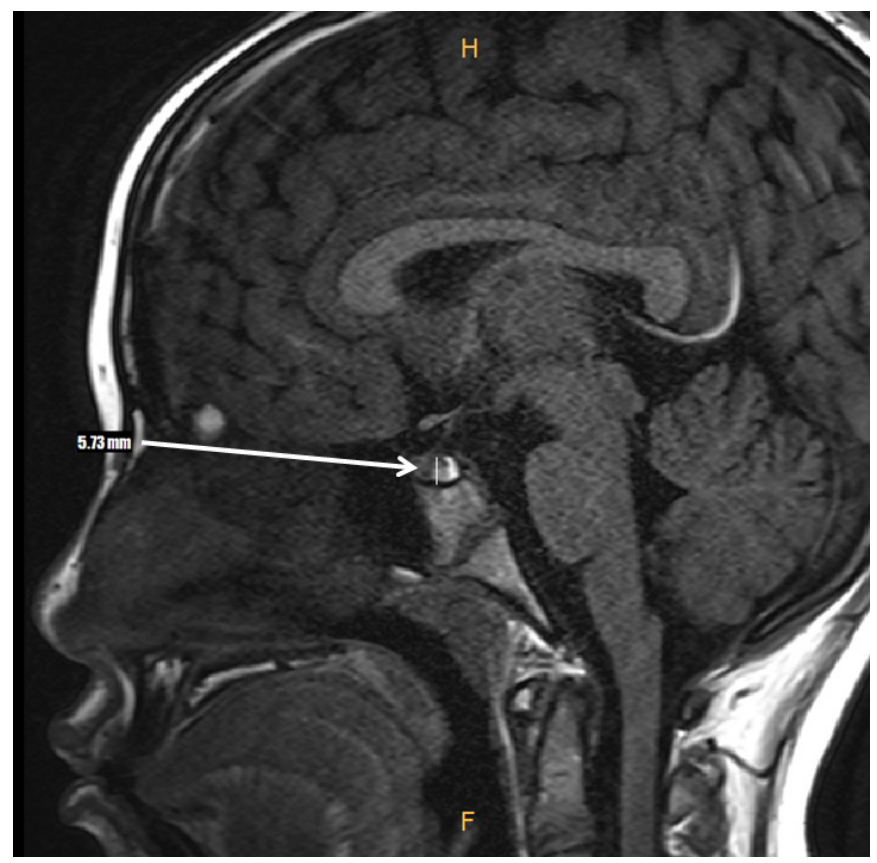

Fig. 2. Brain contrast enhancement T1WI sagittal magnetic resonance imaging. Small cystic lesion (white arrow) appeared between anterior and posterior pituitary gland, representing a small Rathke's cleft cyst.

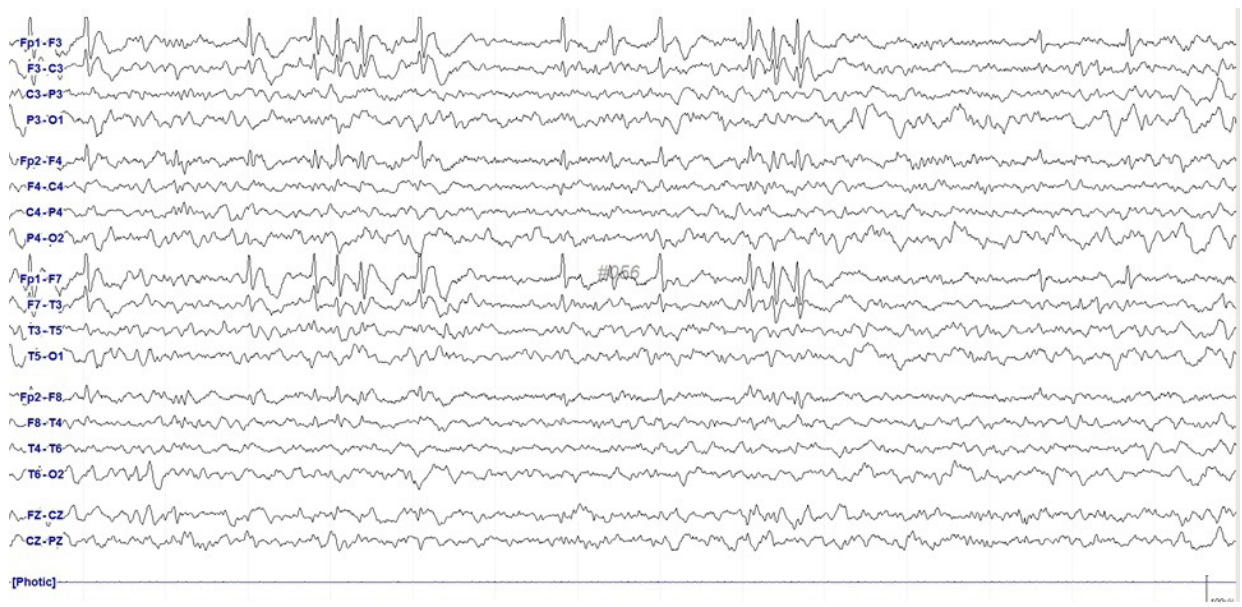

Fig. 1. Electroencephalogram (EEG) of the patient. EEGs showed frequent medium to high voltage spike or sharp wave discharges from left fronto-temporal and right frontal areas. 
characterized with focal seizures restricted to both arms which usually developed as secondary generalized tonic seizures. Serial EEG tests and brain magnetic resonance imaging (MRI) were administered. The EEG results consistently showed moderately abnormal sleep records due to a few small spike discharges, dominantly in the fronto-centro-temporal areas, and she was prescribed anti-epileptic drugs. Oxcarbazepine (Trileptal ${ }^{\circledR} ;$ Novartis international $A G$, Basel, Switzerland), the first drug she tried, was not effective as she continued to have focal seizures, and topiramate (Topamax ${ }^{\circledR}$; Novartis international AG) was therefore added. The frequency of seizures decreased significantly, and she was seizure-free for over two years. Spikes in the EEG were still observed (Fig. 1), leading us to closely monitor her symptoms. An initial brain MRI showed no abnormal findings, but in follow-up, a small Rathke's cleft cyst was found, and was suspected as to be an incidental finding (Fig. 2).

Her developmental was also evaluated. When she was seven years old, her height was $129.0 \mathrm{~cm}$ (50-75 percentile) and weight was $27.2 \mathrm{~kg}$ (25-50 percentile), within normal ranges. However, a slow decline in growth velocity was apparent. A combined pituitary functions test-a method for simultaneously assessing the hypo-function of the anterior pituitary hormones ( $\mathrm{GH}$, adrenocorticotropic hormone [ACTH], follicle-stimulating hormone [FSH], luteinizing hormone [LH], TSH, and prolactin)measured her peak $\mathrm{GH}$ level as $2.2 \mathrm{ng} / \mathrm{mL}$ which was under 10

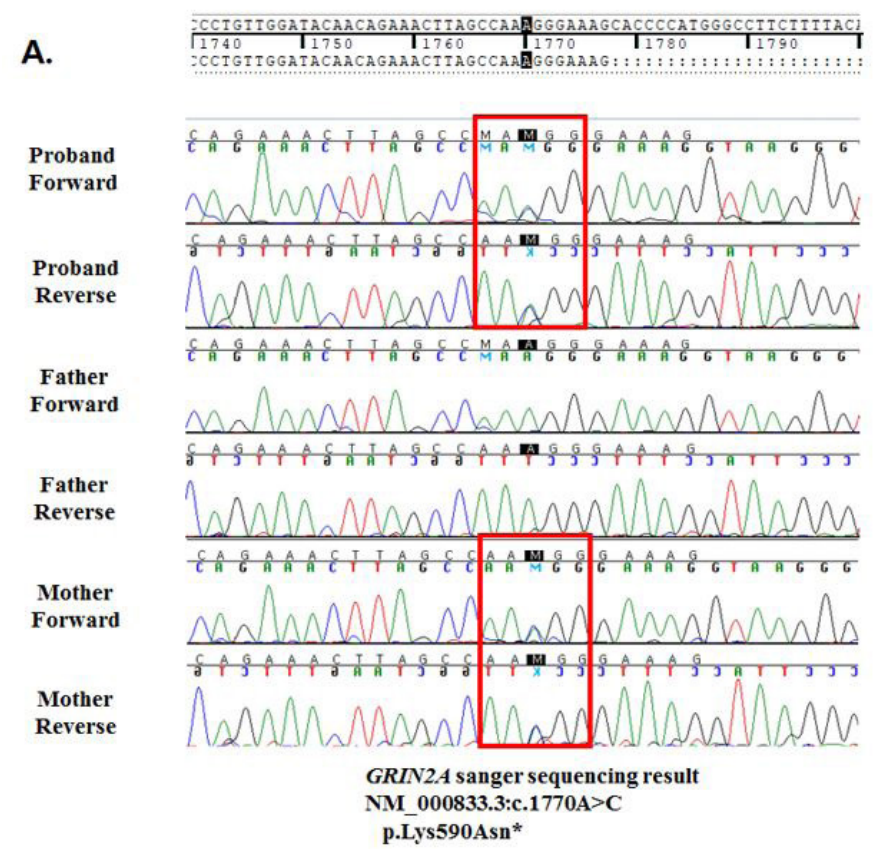

$\mathrm{ng} / \mathrm{mL}$, raising the possibility of a $\mathrm{GH}$ deficiency. Another followup $\mathrm{GH}$ stimulation test with L-dopa and arginine also resulted in a diagnosis of $\mathrm{GH}$ deficiency, and we decided to add recombinant $\mathrm{GH}$ replacement to catch up with her expected growth pattern. Her receptive and expressive language, and social development was also delayed by about two years, although her motor developments met standard milestones and was appropriate for her age. In consultation with a psychiatrist, we found her intelligence quotient to be 71 and memory quotient to be 76 , suggesting borderline intellectual disability.

To investigate further, we carried out genetic analysis using diagnostic exome sequencing of the patient, and Sanger sequencing of her unaffected parents. For diagnostic exome sequencing, genomic DNA was extracted from EDTA whole blood and all exons of 5,447 genes captured using a Celemics G-Mendeliome DES Panel. The post-capture library DNA was subjected to sequence analysis and the sequence reads were mapped and compared with the published human genome build (UCSC GRCh37 or hg 19 reference). We found that the patient has a missense and a nonsense mutation in GRIN2A and neither mutation have previously been reported as related to GRIN2A-related EAS. The missense mutation (c.1770A>C; p.Lys509Asn, likely benign, focal epilepsy with speech disorder and with or without mental retardation, autosomal dominant) was found only in her mother, indicating a high possibility that the variant is benign. The non-

B.
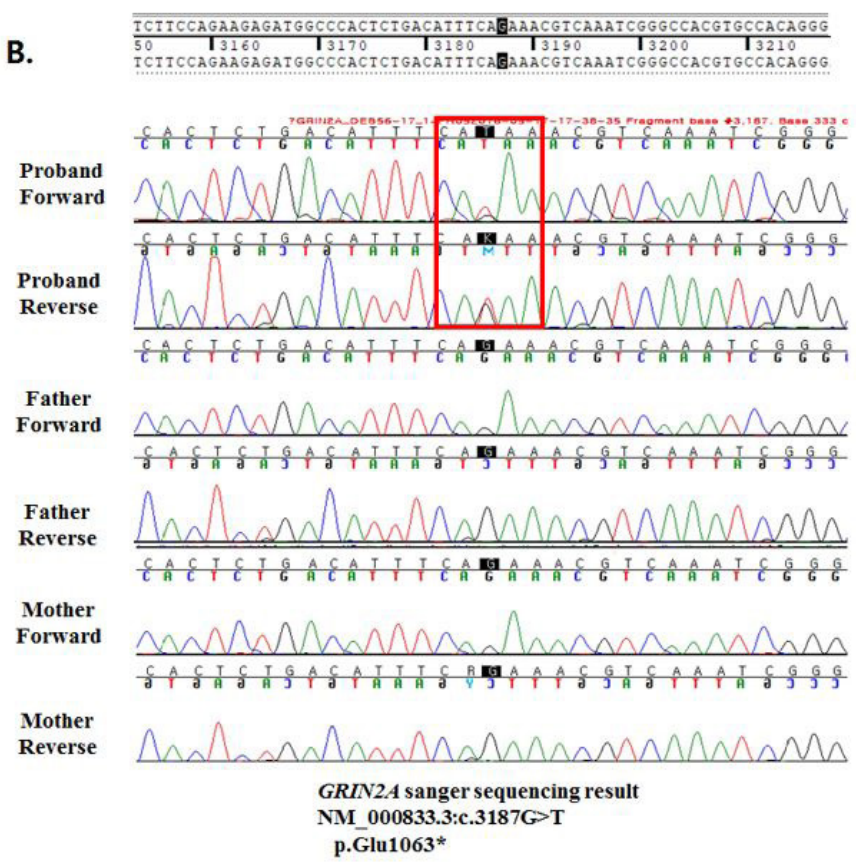

Fig. 3. Sanger sequencing of the proband and her parents. Sequencing showed (A) a missense and (B) a nonsense mutation of the GRIN2A gene (c.1770A>C; p.Lys509Asn and c.3187G>T; p.Glu1063*, respectively). 
sense mutation (c.3187G>T; p.Glu1063, pathogenic, FESD, AD), which was not found in either parent, implying that the mutation was de novo, was considered as pathogenic (Fig. 3). Other genes, including thyroid disease-associated genes, were all found to be normal. Based on these results, we assume that her epilepsy and developmental delay could be related to this mutation, although the relationship between the mutation and other features such as congenital hypothyroidism remains unclear.

\section{Discussion}

EAS disorders, which are characterized by epilepsy and developmental delay, have only been understood as arising from autoimmune system failure [3]. However, with discovery of GRIN2A mutations, the pathophysiology and clinical features of EAS disorders became clearer, and GRIN2A mutations became another wellknown predictive factor in the EAS disorders. GRIN2A is a gene encoding subunit of the NMDA receptors in the brain, NMDA receptors determine synaptic responses, so NMDA receptor-mediated signaling can contribute to numerous brain diseases [3]. Our patient had also been diagnosed with and treated for epilepsy and developmental delay, and detection of the GRIN2A mutations gave us greater insight into the origins of her condition.

The relationships between mutations in this gene and nonneurological symptoms are not well understood, and the testing available for these patients is limited. No phenotypes arising from mutations in GRIN2A, other than those discussed above, are known to be definitely pathogenic. However, this patient had both GRIN2A mutations and permanent congenital hypothyroidism, GH deficiency and Rathke's cleft cyst in the brain. These issues can be treated in isolation, but we could not help but wonder about the relationship between the non-neurologic features and the mutations in GRIN2A. One similar case report of hypothyroidism with mutation in GRIN2B, which also encodes subunits of the NMDA receptors, might shed further light upon this question $[9,10]$. Furthermore, a recently proposed therapy with memantine, a NMDA receptor antagonist discovered by understating the activity of NMDA receptors, would be able to use in patients with non-neurological problems from GRIN2A mutations [11].

By presenting this case, we raise questions about the phenotypic spectrum of GRIN2A-related disorders, embracing broader problems regarding neurological and non-neurological functions. Further studies will be necessary to elucidate the possible non-neurological phenotypes generated by the GRIN2A mutations and their underlying molecular mechanisms.
In conclusion, patients with EAS disorders caused by GRIN2A mutations should be examined for other, non-neurologic features, especially congenital hypothyroidism, as in our patient. We hope the inclusion in the literature of studies of different cases will encourage appropriate genetic counseling for patients, and allow them to be considered candidates for targeted treatment, with positive consequence.

\section{References}

1. Yang $X$, Oian $P, X u X$, Liu $X$, Wu $X$, Zhang $Y$, et al. GRIN2A mutations in epilepsy-aphasia spectrum disorders. Brain Dev 2018;40:205-10.

2. Carvill GL, Regan BM, Yendle SC, O'Roak BJ, Lozovaya N, Bruneau N, et al. GRIN2A mutations cause epilepsy-aphasia spectrum disorders. Nat Genet 2013;45:1073-6.

3. Lesca G, Rudolf G, Bruneau N, Lozovaya N, Labalme A, Boutry-Kryza $N$, et al. GRIN2A mutations in acquired epileptic aphasia and related childhood focal epilepsies and encephalopathies with speech and language dysfunction. Nat Genet 2013;45:1061-6.

4. Lemke JR, Lal D, Reinthaler EM, Steiner I, Nothnagel $M$, Alber $M$, et al. Mutations in GRIN2A cause idiopathic focal epilepsy with rolandic spikes. Nat Genet 2013;45:1067-72.

5. Myers KA, Scheffer IE. GRIN2A-related speech disorders and epilepsy. In: Adam MP, Ardinger HH, Pagon RA, Wallace SE, eds. GeneReviews ${ }^{\circledR}$. Seattle: University of Washington, 2016.

6. Gao K, Tankovic A, Zhang Y, Kusumoto H, Zhang J, Chen W, et al. A de novo loss-of-function GRIN2A mutation associated with childhood focal epilepsy and acquired epileptic aphasia. PLoS One 2017;12:e0170818.

7. Strehlow V, Heyne HO, Vlaskamp DRM, Marwick KFM, Rudolf G, de Bellescize J, et al.; GRIN2A study group. GRIN2A-related disorders: genotype and functional consequence predict phenotype. Brain 2019;142:80-92.

8. Fernández-Marmiesse A, Kusumoto $H$, Rekarte $S$, Roca I, Zhang J, Myers $\mathrm{SJ}$, et al. A novel missense mutation in GRIN2A causes a nonepileptic neurodevelopmental disorder. Mov Disord 2018;33:992-9.

9. Endele S, Rosenberger G, Geider K, Popp B, Tamer C, Stefanova I, et al. Mutations in GRIN2A and GRIN2B encoding regulatory subunits of NMDA receptors cause variable neurodevelopmental phenotypes. Nat Genet 2010;42:1021-6.

10. Ghasemi M, Schachter SC. The NMDA receptor complex as a therapeutic target in epilepsy: a review. Epilepsy Behav 2011;22:617-40.

11. Pierson TM, Yuan $H_{1}$ Marsh ED, Fuentes-Fajardo $K_{1}$ Adams DR, Markello T, et al. GRIN2A mutation and early-onset epileptic encephalopathy: personalized therapy with memantine. Ann Clin Transl Neurol 2014;1:190-8. 